\title{
Essais
}

Revue interdisciplinaire d'Humanités

\section{Écriture épistolaire et éducation humaniste}

\author{
Maria Cristina Panzera
}

\section{OpenEdition}

Journals

Édition électronique

URL : https://journals.openedition.org/essais/9710

DOI : 10.4000/essais. 9710

ISSN : 2276-0970

Éditeur

École doctorale Montaigne Humanités

Édition imprimée

Date de publication : 15 avril 2014

Pagination : 41-52

ISBN : 978-2-9544269-2-1

ISSN : 2417-4211

Référence électronique

Maria Cristina Panzera, «Écriture épistolaire et éducation humaniste», Essais [En ligne], 4 | 2014, mis en ligne le 17 janvier 2022, consulté le 20 janvier 2022. URL : http://journals.openedition.org/essais/ 9710 ; DOI : https://doi.org/10.4000/essais.9710 


\section{Écriture épistolaire et éducation humaniste}

\section{Maria Cristina Panzera}

Écrire à autrui n’a jamais été aussi facile qu’à notre époque : il nous suffit en effet de quelques frappes sur un clavier pour joindre, voire submerger de messages un proche, si ce n'est des inconnus, via mail, fax, sms et toute sorte de réseaux informatiques dont bénéficie désormais notre civilisation de la communication globale dans sa quête permanente, mais sans doute un peu illusoire, de libération de la parole, par-delà les frontières d'espace, de distinction sociale, d'identité culturelle ou linguistique ${ }^{1}$. Liberté, à l'époque humaniste et renaissante, rime, bien que cela puisse nous paraitre étrange aujourd'hui, avec contrainte : cultiver les arts libéraux, ceux qui libèrent l'homme de l'esclavage de l'ignorance et de l'indignité morale, ne peut se faire que dans l'effort, la discipline de soi, le dialogue avec les Anciens, l'intériorisation, enfin, de normes sociales et morales qui associent de manière très étroite la maitrise de la parole, à savoir l'éloquence, et l'excellence des mœurs, selon l'idéal de l'orateur ancien vir bonus dicendi peritus, un homme de bien possédant l'art de la parole. Étant donné que l'écriture épistolaire est considérée, suivant une tradition très ancienne, comme une forme de conversation à distance ${ }^{2}$, elle participe de cette éducation à la parole que l'humanisme s'était donné comme idéal. On comprend, alors, que l'échange épistolaire occupe une place très importante dans les pratiques sociales, politiques et culturelles de l'époque pré-moderne, jusqu'à s'ériger en genre littéraire à part entière, sans doute, aux côtés du dialogue, le genre que les humanistes ont le plus affectionnés.

1 Quelques aspects de la cyber-communication de nos jours rentrent dans l'analyse anthropologique et sociologique proposée par Z. Baumann, L’Amour liquide. De la fragilité des liens entre les hommes, Paris, Hachette, 2004.

2 Le rapprochement entre la lettre et l'art du discours oral constitue un véritable topos des manuels épistolaires depuis le Moyen-Âge. Conrad de Mure expose par exemple cette définition vers 1275 dans son De arte prosandi : "sermo habetur ad presentes, epistola dirigitur ad absentes ", à savoir « le discours s'adresse à un public présent, la lettre à des absents ", cf. G. Gueudet, L'art de la lettre humaniste, Paris, Champion, 2004, p. 229.

3 Sur l'importance du genre épistolaire à la Renaissance P. O. Kristeller, Medieval Aspects of 
Plusieurs savants, pédagogues et hommes de lettres ont participé à l'élaboration de cette culture de l'échange épistolaire dans l'Europe humaniste, à l'image d'un Érasme, pour qui ce genre d'écriture représentait une tâche quotidienne liée à son activité professionnelle ; aussi se plaint-il à plusieurs reprises dans sa correspondance de la quantité de temps qu'il doit consacrer à lire et à écrire des lettres. Il lui arrive, par exemple, comme il le confie à Budé dans une lettre de 1516, de devoir en rédiger une vingtaine en l'espace d'une seule journée 4 .

Un tel élan d'écriture n'est pas, cependant, l'apanage exclusif des hommes de lettres, comme le montre un exemple moins connu tiré des Livres de la famille de Leon Battista Alberti (1443), où l'un des membres de la famille Alberti, Adovardo, est loué comme un modèle de dynamisme justement en vertu des liens de correspondance qu'il est capable d'entretenir :

Ma tu, Adovardo, che se' quanto sia possibile sollecito, che mai fuor di casa ti vidi sì ozioso che tu non sollecitassi le cose di fuori, tutto il dì ti veggo scrivere, mandare fanti a Bruggia, a Barzalona, a Londra, a Vignone, a Rodi, a Ginevra, e d'infiniti luoghi ricevere lettere, e ad infinite persone al continuo rispondere, e fai sì che essendo tu coi tuoi, ancora t'inframetti in molti altri luoghi, e senti e sai quello che per tutto si fa ${ }^{5}$.

Adovardo Alberti est représenté ici en bon père de famille, au centre d'un réseau de correspondance épistolaire très vaste (souligné par les anaphores des adjectifs "infiniti luoghi ", "infinite persone ", ainsi que par l'enchaînement des verbes à l'infinitif "scrivere, ricevere, rispondere ») lui conférant comme un don

Renaissance Learning, Durham, Duke University Press, 1974, et au moins M. Fumaroli, «Genèse de l'épistolographie classique : rhétorique humaniste de la lettre, de Pétrarque à Juste Lipse ", in Revue d'histoire littéraire de la France, LXXVIII, 1978, p. 886-900, A. Quondam, Le " carte messaggiere ". Retorica e modelli di comunicazione epistolare : per un indice dei libri di lettere del Cinquecento, Rome, Bulzoni, 1981, G. Gueudet, L'art de la lettre, op. cit., L. Vaillancourt, La lettre familière au XVI siècle : rhétorique humaniste de l'épistolaire, Paris, Champion, 2003. Pour l'aire italienne en particulier J. Basso, Le genre épistolaire en langue italienne (1538-1622). Répertoire chronologique et analytique, Rome-Nancy, Bulzoni-Presses Universitaires de Nancy, 2 vol., 1990.

4 Désiré Érasme, Opus epistolarum, a c. di P. S. Allen, Oxford, Clarendon, 1906, t. II, p. 363, épître 480, 1. 27. Dans une autre lettre Érasme déclare qu’il doit passer plus de la moitié de son temps à lire et écrire des lettres (Érasme à Henry Bottens, 29 mars 1528, in Opus epistolarum, t. VII, p. 376, 1. 3-4). Selon G. Gueudet, L'art de la lettre humaniste, op. cit., p. 67-68 : " Le temps considérable qu'il doit consacrer à ses échanges, rançon de sa gloire, témoigne de sa prééminence en Europe, mais leur fréquence et leur multiplicité montrent également qu'avec lui une étape a été franchie dans le développement de l'épistolographie ".

5 Leon Battista Alberti, I libri della famiglia, Turin, Einaudi, 1972, p. 85 : « Mais toi Adovardo, qui es plein d'entrain autant qu'on peut l'être, au point que je ne t'ai jamais vu rester désœuvré hors de chez toi sans t'enquérir de ce qui se passe ailleurs, je te vois écrire toute la journée, envoyer tes messagers à Bruges, à Barcelone, à Londres, à Avignon, à Rhodes, à Genève et recevoir des lettres en provenance d'une infinité de lieux, répondre continuellement à un nombre infini de personnes, de sorte que tu restes avec ta famille tout en intervenant dans un grand nombre d'autres lieux et que tu entends et apprends ce qui se fait partout ". 
d'ubiquité. Le bon marchand responsable de la fortune économique de sa famille rejoint en lui l'homme de culture, dont on célèbre l'ouverture sur le monde, la curiosité, la volonté d'être informé de tout ${ }^{6}$. En effet, les Alberti appartiennent à l'une des familles florentines les plus puissantes, de banquiers et d'humanistes, qui s'était toujours illustrée dans le domaine intellectuel, comme l'auteur le souligne dans d'autres passages de son traité, précisant que « nelle lettere fu sempre eccellentissima ", à savoir qu'elle fut toujours excellente dans les Belles Lettres.

Cette osmose entre deux modèles culturels différents, ici l'esprit d'entreprise des marchands et l'idéal humaniste de la vie active, prouve à quel point il serait difficile, sur le plan méthodologique, de proposer des distinctions trop nettes entre les différentes formes d'écriture épistolaire, par exemple entre correspondance humaniste en latin, lettres diplomatiques ou de chancellerie, lettres commerciales et marchandes, ou encore lettres de famille et privées en langue vernaculaire ${ }^{7}$. Nombre de questions restent en particulier encore ouvertes au sujet du socle rhétorique commun à la base de toutes ces écritures, allant de l'enseignement de l'art de la lettre jusqu'à l'étude des textes manuscrits ou imprimés. Ceci malgré le renouveau considérable des recherches dans le domaine épistolaire qui s'est produit depuis les années soixante-dix et quatre-vingt et qui a marqué le passage d'une approche de type positiviste, où la lettre était considérée comme une simple source d'informations au service de l'histoire événementielle et de la prosopographie, à une approche sémiologique prenant en compte les fonctions communicationnelles et performatives du discours épistolaire, son rapport à la société et à l'histoire des mentalités. Ce changement d'orientation concerne aussi bien la littérature que l'histoire, étant donné les caractéristiques interdisciplinaires d'un tel domaine de recherche. En littérature, on s'intéresse en particulier aux stratégies d'autoreprésentation et d'autopromotion des hommes de lettres à travers la publication de leurs épistolaires, mais également aux rapports de pouvoir et aux politiques culturelles liées au marché de l'imprimerie ${ }^{8}$. En histoire, l'étude des

6 Cette capacité à centraliser toute sorte d'informations peut avoir également des implications politiques, par exemple dans le même traité d'Alberti au livre IV, p. 334 le vieux Piero Alberti raconte comment il a pu devenir le protégé du Duc de Milan, Giangaleazzo Visconti : il disposait d'un réseau de relations dans toute la Méditerranée et il était par conséquent informé de tout " o tumulti, armate, esserciti o legge nuove, affinità tra principi, publice amicizie, armi o incendii, naufragi o qualunque cosa accadesse per le provincie nuova e degna di memoria, subito me ne faceano certo ", il était donc un parfait conseiller pour le Duc.

7 Dans son livre sur la fonction politique de la correspondance entre Machiavel et Vettori, John Najemi propose la distinction opératoire entre lettres privées en langue vernaculaire, lettres diplomatiques ("utilitarian letters ») et lettres des humanistes, mais il signale les limites d'une classification trop rigide, voir J. Najemi, Between friends. Discourses of Power and Desire in the Machiavelli-Vettori Letters of 1513-1515, Princeton, Princeton University Press, 1993.

8 A. Quondam, Le "carte messaggiere", op. cit., R. Morabito, Lettere e letteratura, Turin, Edizioni dell'Orso, 2001 ; R. Rinaldi, L'epistolario moltiplicato, in Storia della civiltà letteraria italiana, sous la direction de G. Barberi Squarotti, vol. II, Umanesimo e Rinascimento, Turin, UTET, 
réseaux de correspondances permet d'appréhender les mécanismes de gouvernement et de domination - clientélisme, administration, diplomatie, etc. -, mais aussi les pratiques sociales et les représentations ${ }^{9}$. Une autre direction de recherche concerne, enfin, les livres de lettres en langue vernaculaire et leur rôle dans la diffusion des idées religieuses en période de Contre Réforme ${ }^{10}$.

Dans toutes ces recherches, l'essor de l'épistolographie renaissante est souvent mis en relation avec une pluralité de facteurs, comme le développement des structures étatiques, de la chancellerie, de la diplomatie, mais également l'invention de l'imprimerie, les changements dans les conditions professionnelles des hommes de lettres, les exigences d'un nouveau public de lecteurs, etc. Afin de mieux évaluer le facteur intellectuel qui participe à l'ensemble de ces changements, nous proposons de mettre l'accent sur les valeurs que les humanistes associent à l'écriture épistolaire : comment et dans quelle mesure les échanges épistolaires permettent-ils de traduire en pratique, dans un programme éducatif défini, le projet humaniste de renouvellement de l'homme et des relations humaines ? Le point de départ est marqué, on le sait, par Pétrarque et par sa redécouverte du genre épistolaire de l'Antiquité. Dans un deuxième temps, il nous faudra analyser la place que l'écriture épistolaire occupe au sein de la pédagogie humaniste.

\section{Pétrarque : du culte de l'Antiquité à la quête de la gloire}

La redécouverte de l'Antiquité et le classicisme qui en découle sont les facteurs qui permettent d'introduire une distinction entre la tradition médiévale de la lettre, telle qu'elle fut développée par les artes dictaminis depuis le XII ${ }^{\mathrm{e}}$ siècle, et l'épistolographie humaniste ${ }^{11}$. Une réflexion nouvelle sur la nature et sur les

1993, p. 1738-75 ; N. Longo, Letteratura e lettere. Indagine nell'epistolografia cinquecentesca, Rome, Bulzoni, 1999 ; M. L. Doglio, L'arte delle lettere. Idea e pratica della scrittura epistolare tra Quattro e Seicento, Bologne, Il Mulino, 2000.

9 J. Boutier, S. Landi, O. Rouchon (éd.), La politique par correspondance. Les usages politiques de la lettre en Italie (XIV-XVIII siècle), Presses Universitaires de Rennes, 2009. Voir également F. Bethencourt et F. Egmond (éd.), Cultural exchange in early modern Europe, vol. III : Correspondence and Cultural Exchange in Europe 1400-1700, Cambridge/New York, Cambridge University Press, 2007.

10 A. J. Schutte, The Lettere Volgari and the Crisis of Evangelism in Italy, "Renaissance Quarterly ", XXVIII, 1975, p. 639-88, P. Simoncelli, Evangelismo italiano del Cinquecento. Questione religiosa e nicodemismo politico, Rome, Istituto Storico Italiano per l'Età Moderna, 1979 et L. Braida, Libri di lettere. Le raccolte epistolari del Cinquecento tra inquietudini religiose e "buon volgare", Rome/Bari, Laterza, 2009.

11 A. Boureau, La norme épistolaire, une invention médiévale, in R. Chartier (éd.), La Correspondance. Les usages de la lettre au XIXe siècle, Paris, Fayard, 1991, p. 127-157, M. Camargo, Ars dictaminis, Ars dictandi, Turnhout, Brepols, 1991 (Typologie des sources du Moyen Âge occidental, 60). L'un des premiers maîtres d'ars dictaminis fut Albéric de Mont-Cassin, mort vers 1105. En Italie la lettre, envisagée comme une écriture publique, connait au XII siècle un rayonnement exceptionnel à partir de deux centres politiques majeurs : la curie romaine et la cour méridionale de Frédéric II, avec son élite de chanceliers, de notaires, entre autres le célèbre Pierre de la 
fonctions de la parole en société se développe entre le XIV et le $\mathrm{XV}^{\mathrm{e}}$ siècles au rythme des différentes acquisitions philologiques, d'abord avec la découverte des manuscrits des lettres de Cicéron, ainsi que de plusieurs de ses discours publics, plus tard avec la remise en circulation des lettres de Pline le Jeune, du traité sur l'art oratoire de Quintilien ou des ouvrages byzantins qui seront récupérés plus tardivement (Demétrius, Libanius, etc.). On connaît l'effet de déclencheur produit sur Pétrarque par sa découverte en 1345 à Vérone, dans la Bibliothèque du Chapitre de la cathédrale, d'un manuscrit ancien contenant les lettres de Cicéron à Atticus, à Brutus et à son frère Quintus. Quelques années plus tard, à Vaucluse, Pétrarque s'attache à l'organisation de ses propres recueils épistolaires dont le premier sera achevé et publié en 1366, le Rerum familiarum liber ("Livre de choses personnelles "), tandis que le second, le recueil des Seniles, à savoir les lettres de la vieillesse, l'accompagnera jusqu'au seuil de la mort ${ }^{12}$.

L'adoption du modèle cicéronien ainsi que des Lettres à Lucilius de Sénèque vise, chez Pétrarque, le dépassement de la tradition médiévale de la scolastique qui avait pourtant produit nombre de formulaires et d'enseignements techniques sur l'écriture épistolaire. Non seulement le latin barbare des dialecticiens et des rhétoriciens de la scolastique lui apparaît déplorable, mais l'art médiéval de la lettre avait ignoré la forte composante humaine, philosophique et morale de la tradition classique. Entre livre de mémoires et recueil de maximes morales, introspection et témoignage historique, les lettres de Pétrarque composent un genre littéraire nouveau qui n'est pas encore le journal intime au sens moderne, mais qui place au centre de l'écriture l'homme et ses passions, en particulier la définition de l'homme de culture, le sage, car comme l'observe bien Ugo Dotti dans son introduction à l'édition française de Familiares : "Pétrarque entendit avant tout brosser un portrait de son existence d'intellectuel $»^{13}$.

Du point de vue de la rhétorique, Pétrarque épistolier recherche la simplicité, la clarté d'expression et le naturel, loin de tout élan oratoire, car il reprend de ses modèles, aussi bien de Cicéron que de Sénèque, la conception de la lettre comme un substitut de la conversation entre amis, ainsi qu'il l'explique à son correspondant Louis de Béringen dans la lettre qui sert de prologue à l'ensemble des Familiares:

Vigne. L'influence des artes dictaminis s'étend sur plusieurs siècles avec une étonnante continuité jusqu'aux manuels de type "néo-scolastique » du milieu du XVI e siècle.

12 F. Petrarque, Lettres familières, t. I-V, livres IV-XIX, Notices et notes de Ugo Dotti, traduction de A. Longpré, Paris, Les Belles Lettres, 2002-2005, id., Lettres de la vieillesse, t. I-IV, livres I-XV, édition critique d'Elvira Nota, traduction de F. Castelli, F. Fabre, A. de Rosny, L. Schebat, P. Laurens, J.-Y. Boriaud, Paris, Les Belles Lettres, 2002-2006, id., Sans titre, Liber sine nomine, 1342-1361, traduit par R. Lenoir, Grenoble, Jérôme Millon, 2003 (une nouvelle traduction est en préparation aux Belles Lettres). Les Familiares comprennent XXIV livres, soit 350 épîtres : c'est le livre que Pétrarque dédie à son ami Ludovic de Beringen désigné par le pseudonyme ou surnom littéraire de Socrate. Les lettres de la vieillesse en XVII livres comptent 125 lettres dédiées à Francesco Nelli (sous le nom de Simonides).

13 F. Pétrarque, Lettres familières, p. XLVI. 
Tu liras alors avec bienveillance et tu jugeras favorablement, comme mes autres œuvres, ces écrits d'un style simple, intime et familier, approprié et conforme aux tournures que nous employons dans la conversation de tous les jours ${ }^{14}$.

Ce recours à une langue de tous les jours, le sermo communis, n'implique pas seulement la simplification du protocole médiéval, par exemple dans les formules de salutation, mais cache en réalité une très grande ambition de la part de l'intellectuel nourri de prose latine : renouer avec les Anciens c'est en effet s'approprier leur façon de penser et même de parler, c'est-à-dire redonner au latin le statut d'une langue maternelle, dans le but de réincarner un modèle de vie et de culture que l'on veut encore actuel. C'est encore Pétrarque qui décrit son rapport à la littérature ancienne comme une rumination, une assimilation tellement profonde qu'il en arrive à oublier ses sources :

J'ai lu Virgile, Horace, Boèce et Cicéron ; je ne les ai pas lus qu'une fois, mais mille, je ne les ai pas parcourus mais couvés, et je m’y suis arrêté, usant de toutes les forces de mon esprit ; je les ai mangés le matin pour les digérer le soir, je les ai avalés dans mon enfance pour les ruminer dans ma vieillesse. Leurs mots se sont installés si intimement en moi, se sont fixés non seulement dans ma mémoire mais dans ma moelle pour ne faire qu'un avec mon esprit, au point que, sans que je les lise davantage tout au long de ma vie, ils resteront en moi, car ils ont pris racine au fond de mon être ; et parfois j'en oublie l'auteur parce que, suite à une longue pratique et à une imprégnation permanente, je les ai comme écrits avant lui et pris pour miens depuis longtemps si bien qu'assiégé par cette foule, je ne me rappelle plus de qui ils sont, ni même s'ils sont de moi ou d'un autre ${ }^{15}$.

Point d'orgue de ce culte de l'Antiquité, l'écriture épistolaire représente aussi, pour Pétrarque, le gage de sa gloire future. La réflexion sur la gloire se dégage tout particulièrement dans la conclusion de la première lettre des Familiares où Pétrarque, après avoir demandé à Louis de Beringen de ne pas divulguer ses lettres tant que le recueil ne sera pas terminé, ajoute :

Mais si un jour je donne la dernière touche non pas, comme dit Cicéron, à la Minerve de Phidias, mais à cette image de mon âme, quelle qu'elle soit, et à ce portrait de mon talent que je m'efforce de polir avec soin, alors, lorsque tu l'auras en tes mains, tu pourras le placer sans crainte aussi en vue que tu voudras ${ }^{16}$.

14 Ibid., I, 116.

15 Ibid., XXII, 2, 12-13 (vol. 4, 106) : "Legi apud Virgilium apud Flaccum apud Severinum ; nec semel legi sed milies, nec cucurri sed incubui, et totis ingenii nisibus immoratus sum ; mane comedi quod sero digererem, hausi puer quod senior ruminarem. Hec se michi tam familiariter ingessere et non modo memorie sed medullis affixa sunt unumque cum ingenio facta sunt meo, ut etsi per omnem vitam amplius non legantur, ipsa quidem hereant, actis in intima animi parte radicibus, sed interdum obliviscar auctorem, quippe qui longo usu et possessione continua quasi illa prescripserim diuque pro meis habuerim, et turba talium obsessus, nec cujus sint certe nec aliena meminerim ».

16 Lettres familières I, 1, 37: «Illam vero non Phidie Minervam, ut ait Cicero, sed qualemcunque animi mei effigiem atque ingenii simulacrum multo michi studio dedolatum, si unquam supremam illi manum imposuero, cum ad te venerit, secure qualibet in arce constituito ". 
Cette visibilité, que Pétrarque souhaite acquérir avec tant d'application, n'est pas confiée à un portrait physique, mais à l'image peinte de sa beauté d'âme et d'esprit, métaphore de l'œuvre littéraire. Selon la note de commentaire fournie pour l'interprétation de ce passage par Ugo Dotti, fin connaisseur de l'œuvre de Pétrarque, l'auteur annoncerait ici un projet autobiographique : "Pétrarque fait allusion à la composition d'un autoportrait en prose dans lequel certains ont voulu voir le projet de l'épître Posteritati ${ }^{17}$. Pour la référence à la statue de Minerve de Phidias, Dotti signale opportunément le passage du deuxième livre du De oratore, où Cicéron compare l'orateur parvenu au sommet de l'art de l'éloquence au célèbre sculpteur grec ${ }^{18}$. Il n'a pas été remarqué, cependant, que les métaphores tirées de la peinture et de la sculpture dans cette première lettre des Familiares (animi mei effgiem atque ingenii simulacrum) renvoient à un autre intertexte cicéronien, le discours Pro Archia de Cicéron, dont on connaît l'importance pour l'élaboration de la conception pétrarquienne de la gloire sur le modèle des Anciens ${ }^{19}$. En célébrant la poésie et la dignité des poètes, Cicéron avait dit dans ce discours que les grands hommes cherchent à se faire représenter dans des statues et des portraits qui en assurent la mémoire, mais que plus importante encore, car éternelle, est la mémoire assurée par l'œuvre littéraire, la seule capable de transmettre à la postérité l'image intérieure de l'homme :

Eh quoi ! si beaucoup d'hommes éminents ont tenu à laisser derrière eux des statues et des images, reproduction non point de leur âme, mais de leur corps, ne devons-nous pas préférer de beaucoup laisser derrière nous le portrait de notre activité et de nos vertus, tracé et parfait par d'éminents génies? Quant à moi, dans les actes que j'accomplissais, dans le moment même où je les accomplissais, je m’imaginais les répandre et les propager pour l'éternité dans le souvenir de l'univers ${ }^{20}$.

Sur cette première lettre des Familiares voir en particulier les commentaires de E. Wolff, "Pétrarque et le genre épistolaire : réflexions sur Familiares I, 1 », in Epistulae Antiquae II. Actes $d u I I{ }^{e}$ Colloque international : Le genre épistolaire antique et ses prolongements européens, Tours, 28-30 septembre 2000, Louvain, Peters, 2002, p. 379-385.

17 Ibid., note 40, p. 350.

18 Cicéron, De l'orateur. Texte établi et traduit par E. Courbaud, Paris, Les Belles Lettres, 1966, livre II, 17, 73 : «Si l'orateur, embrassant ces diverses parties de sa tâche, parvient, nouveau Phidias, à dresser en pieds une Minerve, alors, soyons-en sûrs, pas plus que le maitre-statuaire n'eut de peine à sculpter le bouclier de la déesse, il n'aura besoin de leçons, lui, pour réaliser des ouvrages de moindre importance ».

19 Pour la conception de la gloire chez Pétrarque S. Verhulst, " Fonction sociale de la poésie au Quattrocento ", in Poétiques de la Renaissance, P. Galand-Hallyn et F. Hallyn (éd.), Genève, Droz, 2001, p. 346-360.

20 Cicéron, Pro Archia XII, 30 (Discours. Pour le poète Archias, texte établi et traduit par F. Gaffiot, t. XII, Paris, Les Belles Lettres, 1959, p. 49) : «An statuas et imagines, non animorum simulacra sed corporum, studiose multi summi homines reliquerunt, consiliorum relinquere ac virtutum nostrarum effigiem nonne multo malle debemus, summis ingeniis expressam et politam ? Ego vero omnia quae gerebam, iam tum in gerendo spargere me ac disseminare arbitrabar in orbis terrae memoriam sempiternam. Haec vero sive a meo sensu post mortem 
Pétrarque ne reprend pas seulement de Cicéron l'idée que l'écrit représente un reflet de l'âme humaine, mais il en développe également les images à travers des reprises lexicales précises : " non animorum simulacra sed corporum " selon l'expression de Cicéron et "virtutum nostrarum effigiem ». L'opposition entre la statue de Minerve de Phidias et la représentation de soi-même que l'homme de lettres confie à l'œuvre littéraire prend donc tout son sens.

La référence au discours cicéronien sur la gloire des orateurs n'apparait pas pour la première fois dans cette première lettre du recueil des Familiares. Elle avait déjà nourri la réflexion de Pétrarque lors de sa présentation de la Collatio laureationis, le célèbre discours prononcé à l'occasion de son couronnement poétique au Capitole de Rome en $1341^{21}$. C'est précisément cette conception de la gloire et de la dignité de l'intellectuel que Pétrarque a légué à la Renaissance. Pierre Mesnard avait donc sans doute raison quand il disait que le commerce épistolaire est l'expression de l'individualisme de la Renaissance. Néanmoins, il faut considérer aussi que la correspondance suppose une communauté de destinataires, qui ne forment pas uniquement un public passif. Pour que cette collectivité se forme et que les valeurs soient largement partagées, il fallait enfin traduire l'idéal humaniste en un projet éducatif à part entière ${ }^{22}$.

\section{La place de l'écriture épistolaire dans la pédagogie humaniste}

L'exemple de Pétrarque a contribué profondément à la fortune des recueils de correspondance en tant que genre littéraire à l'époque humaniste : plusieurs humanistes, de Coluccio Salutati à Leonardo Bruni en passant par Ange Politien, ont attaché une attention particulière à leur propre production de lettres en langue latine et se sont illustrés comme modèles de prose épistolaire ${ }^{23}$. Mais c'est au sein même de la pédagogie humaniste que l'écriture épistolaire finit par conquérir une place importante, particulièrement chez

afutura est sive - ut sapientissimi homines putaverunt - ad aliquam mei partem pertinebit, nunc quidem certe cogitatione quadam speque delector $"$.

21 S. Verhulst, "Fonction sociale de la poésie ", op. cit. Dans la Collatio Laureationis, Pétrarque cite deux fois de manière explicite le Pro Archia de Cicéron, qu'il avait lui-même découvert à Liège en 1333. À la page 351 de son article, S. Verhulst analyse un passage de la lettre de Pétrarque à Thomas de Messine au sujet de la gloire dont il est dit qu'elle ne peut être que posthume (Familiares I, 2, 18) et y reconnaît le renversement d'un motif présent dans le Pro Archia. Elle ne signale pas la référence au Pro Archia dans Fam. I,1.

22 P. Mesnard, "Le commerce épistolaire comme expression de l'individualisme humaniste ", Individu et société à la Renaissance, Paris/Bruxelles, Presses universitaires de France/Presses universitaires de Bruxelles, coll. "Travaux de l'Institut pour l'étude de la Renaissance et de l'humanisme ", 1967, p. 17-31.

23 C. Griggio, Dalla lettera all'epistolario. Aspetti retorico-formali dell'epistolografia umanistica, in A. Chemello (éd.), Alla lettera. Teorie e pratiche epistolari dai Greci al Novecento, Milan, Guerini Studio, 1998. 
les maîtres de l'Italie du Nord dans le premier tiers du XV siècle ${ }^{24}$. Dans les écoles de Gasparino Barzizza, Guarino Veronese, Vittorino de Feltre les programmes d'étude, fixés dans le curriculum, font une place à la lecture des auteurs de l'Antiquité pour les élèves dès leur plus jeune âge. Même si les vieux manuels élaborés par l'enseignement médiéval, comme l'Ars minor de Donat ou les Disticha Catonis, continuent d'être utilisés, les maîtres humanistes se mettent à préconiser la lecture précoce et directe des œuvres de Virgile et de Cicéron. Guarino en arrive même à recommander la mémorisation des lettres de Cicéron et à baser l'enseignement du latin dans son école (fondée en 1390) sur la prose des lettres plutôt que sur la prose philosophique, car il la juge plus accessible et plus agréable pour les apprenants ${ }^{25}$. Une anthologie de cinquante lettres de Cicéron servait de texte d'étude dans son école. À la fin du XVI siècle, les programmes d'étude de certains collèges anglais prévoient la lecture des Lettres familières de Cicéron pour les enfants de dix ans ${ }^{26}$. Enfin, dans son traité Della istitutione morale, publié à Venise en 1560, Alessandro Piccolomini affirme que les enfants peuvent lire les $\mathrm{Ad}$ familiares et en tirer beaucoup de profit ${ }^{27}$. Le principe de l'assimilation de l'éloquence et du style à travers la lecture précoce des auteurs est donc la clé de voûte de cette formation humaniste réservée principalement à l'élite, aux enfants des familles nobles et futurs cadres de l'administration, même si les données quantitatives concernant l'organisation de l'instruction scolaire dans les principales villes italiennes de la Renaissance témoignent d'une certaine diffusion de la scolarisation, y compris parmi les classes moyennes ${ }^{28}$.

24 P. Grendler, Schooling in Renaissance Italy. Literacy and Learning 1300-1600, Baltimore and London, The Johns Hopkins University Press, 1989, p. 133 : "Humanistic education became established in the 1430s, 1440s and 1450s, especially in towns within the orbit of Guarino et Vittorino. The studia humanitatis soon spread throughout northern and north-central Italy ».

25 Guarino accorde une place importante à Cicéron dans son curriculum pédagogique quand il enseigne à Venise entre 1414 et 1419 et il est l'auteur d'un commentaire à Pro Roscio Amerino dont Poggio Bracciolini avait retrouvé un manuscrit en 1415. Lors de l'ouverture de son école à Vérone, en 1390, il dit qu'il a choisi les lettres de Cicéron comme corpus d'enseignement plutôt que les discours qui offrent une prose plus difficile : "Nonnullas enim decerpsi epistulas in quibus ille puri et facetissimi sermonis stilus exprimitur ", cf. R. Sabbadini, Le scoperte dei classici latini e greci nei secoli XIV e XV, Florence, Sansoni, 1914, p. 58.

26 Voir la préface de C. Margolin à son édition de D. Érasme, Opus de conscribendis epistolis, in Opera omnia, Amsterdam, North Holland Publishing Company, vol. I-II, 1971, p. 157-579, ici p. 191.

27 Alessandro Piccolomini, Della institutione di tutta la vita de l'homo nato nobile e in città libera, libri X, Venezia, Gerolamo Scoto, 1545, p. 109.

28 E. Garin, L'educazione in Europa (1400-1600) : problemi e programmi, Bari, Laterza, 1957, P. Grendler, Schooling in Renaissance Italy, op. cit., R. Black, Humanism and Education in Medieval and Renaissance Italy, Cambridge University Press, 2001. Sur la base du cadastre de Florence de 1480 Grendler a calculé que 1031 enfants âgés de 4 à 17 ans fréquentaient l'école : "Approximately 28 percent of boys aged 10 through 13 attended formal schools in Florence in 1480 », P. Grendler, Schooling in Renaissance Italy, op. cit., p. 77, mais il ajoute : " A somewhat 
De l'assimilation par la lecture, on passait à l'imitation active des auteurs dans une phase plus avancée de la scolarité qui correspond à l'étude de la rhétorique, entre douze et dix-huit ans. Guarino Veronese est peut-être le premier à introduire dans son école l'écriture des lettres en tant qu'exercice pédagogique $^{29}$. Le maître proposait un sujet en langue vernaculaire et les étudiants devaient composer en latin. Un autre exercice, le thème, consistait à traduire une lettre en latin à partir d'un modèle en langue vernaculaire proposé par le maître. Une fois son devoir corrigé, l'étudiant le recopiait au propre dans un cahier qu'il conservait chez lui, car il pouvait constituer un répertoire utile en toutes circonstances.

Un renouveau des méthodes et des manuels concerne également l'enseignement universitaire à la faculté des Arts ou celui qui était dispensé dans les écoles de chancellerie, par exemple à Florence, à Milan ou à Venise. Nombre de traités publiés en latin ou en langue vulgaire entre le XV et le XVII siècles témoignent de cet engouement pour l'épistolographie ${ }^{30}$. Il en ressort un certain nombre de contraintes et de normes qui régissent le genre épistolaire. L'apprenti est appelé, par exemple, à respecter la distinction canonique du protocole de la lettre en cinq parties salutatio, exordium, narratio, petitio, conclusio, outre le critère de la convenientia, à savoir le fait d'adapter le message à son destinataire, aux circonstances et aux buts de la communication, selon la formule " quis, cui, quomodo, quando " (le sujet qui écrit, le destinataire, les modalités et les circonstances d'écriture). Pour la partie initiale, la salutatio, des listes de titres honorifiques sont proposées en fonction des différents rangs des interlocuteurs potentiels. Les manuels les plus complets proposent en outre une typologie des lettres selon leur fonction pragmatique, allant de la louange au blâme, à la recommandation, etc. L'un des traités les plus exhaustifs dans ce domaine, celui de Jean-Marie Philelphe, arrive à compter jusqu'à quatre-vingts types différents de lettres ${ }^{31}$.

Par-delà l'aspect rhétorique, c'est toute une représentation des liens sociaux qui entre en jeu dans ces traités, car il s'agit pour l'apprenti épistolier d'assimiler un code de comportement fondé sur l'éthos cicéronien de l'amitié qui le prépare à agir et à s’insérer de manière efficace dans la société. En ce sens, il a été remarqué

conservative overall literacy (reading and writing) estimate of 30 percent to 33 percent seems reasonable for Florentine male population in 1480 ». Pour Venise, le calcul se fonde sur la «Profession de foi » de 1587 demandée aux enseignants par le pape Pie IV. On trouve à l'école 4595 garçons et 30 filles. En tout, cela représente 33 \% des garçons (ibid., p. 46). Le nombre d'enseignants dans le cursus de latin s'élève à 188. Ils sont 258 dans l'enseignement en langue vulgaire, mais nous savons très peu de choses quant à leurs méthodes d'enseignement.

29 P. Grendler, Schooling in Renaissance Italy, op. cit., p. 231.

30 J. Basso, Le genre épistolaire en langue italienne, op. cit., et C. H. Clough, "The Cult of Antiquity: Letters and Letters Collections ", in Cecil H. Clough (éd.), Cultural Aspects of the Italian Renaissance, Manchester, Manchester University Press, 1976, p. 33-67, avec une liste de traités.

31 Gian Mario Filelfo, Novum epistolarium, per Bacilerium de Bacileriis, 1489. 
que la fortune des traités sur l'art épistolaire correspond, à l'époque renaissante, à l'essor des traités de comportement, dans le sillage du Galateo ou du Cortigiano de Baltazar Castiglione. Cet aspect normatif concernant les comportements en société ressort bien, pour citer un exemple concret, du Formulario de epistole vulgare attribué à Cristoforo Landino, publié la première fois en 1485, dont nous pouvons citer quelques paragraphes significatifs :

5. Exorde et excuses lorsque l'on a négligé un ami qui nous a procuré un grand bénéfice.

6. Exordes et excuses lorsque l'on n'a pas pris soin d'écrire à un ami de condition meilleure que la nôtre.

7. Par quelles expressions douces et honnêtes on peut se procurer l'amitié d'un homme de bien.

28. Comment tu peux répondre lorsque quelqu'un te recommande à l'un de ses chers amis ou à un membre de sa famille ${ }^{32}$.

Prise en compte des hiérarchies sociales, apprentissage des bonnes manières, quête d'alliances et de protections dans le respect de l'honestum et du decorum, l'éventail des fonctions performatives que l'épistolier apprend à maîtriser est le gage de sa propre réussite en société et de celle de sa famille. Désormais, c'est la rhétorique épistolaire en langue vernaculaire qui reprend le flambeau, dans une ligne de continuité dont témoigne le célèbre Del Secretario de Francesco Sansovino, un autre succès éditorial équivalent à celui de Landino, lancé à Venise en 1564 et destiné à influencer profondément l'enseignement de l'art épistolaire dans l'Europe de la Renaissance ${ }^{33}$.

De Pétrarque à ses continuateurs, la réforme humaniste de la culture a su exploiter tout le potentiel éducatif de l'échange épistolaire. Elle parvient à dé-monopoliser les savoirs que l'université médiévale marquée par la scolastique avait soumis aux contraintes rigides de l'aristotélisme réduit à l'inflation de la logique et de la dialectique. L'écriture épistolaire permettait en effet aux hommes

32 C. Landino, Formulario de epistole vulgare, Bologne, Ugo di Rugerij, 1485. Selon R. Cardini "Ce fut pendant longtemps, jusqu'au milieu du XVI" siècle, l'un des best-sellers de la bourgeoisie non humaniste et des fonctionnaires de l'administration comme de la diplomatie des Seigneuries et des Principautés de l'Italie de l'époque ", cf. Landino Cristoforo, Scritti critici e teorici, a c. di R. Cardini, Rome, Bulzoni, 1974, p. 179.

33 V. Mellinghoff-Bourgerie, "Le Secrettaire de Gabriel Chappuys face au Del Secretario de Francesco Sansovino et à The English Secretary d'Angel Day. Remarques sur l'héritage de l'éthos épistolographique érasmien ", in Il segretario è come un angelo : trattati, raccolte, epistolari, vite paradigmatiche, ovvero come essere un buon segretario nel Rinascimento, a cura di Rosanna GorrisCamos, Fasano, Schena Ed., 2008, p. 63-92 et L. Braida, Libri di lettere, op. cit., p. 201-18. Pour le rapport de Sansovino avec le traité d'Érasme voir M. C. Panzera, «Francesco Sansovino lecteur d'Érasme. Le De conscribendis epistolis dans la formation du bon secrétaire ", Bibliothèque d'Humanisme et Renaissance, LXXIV, 2012, n 1, p. 83-101. Nous avons signalé les sources humanistes de Sansovino, in M. C. Panzera, "Sansovino e l'umanesimo veneziano. La fonte nascosta dei modelli di lettere del Del Secretario ", Italianistica, XLI, n 2, 2012, p. 21-48. 
de lettres de pratiquer une forme de culture plus humaine et plus proche de la vie, même si elle reposait en grande partie sur l'assimilation d'un ensemble de normes et de modèles faisant autorité et tirés du passé. On trouve en effet, à la base de cette pédagogie, une conception de la civilisation comme construction d'une identité collective et il faudra attendre le XIX ${ }^{\mathrm{e}}$ siècle pour que l'éducation soit établie davantage sur une philosophie du naturel. En s'interrogeant sur les facteurs qui ont assuré à la culture humaniste son vaste rayonnement, Francisco Rico a insisté sur sa flexibilité et sa plasticité, car cette culture avait pour vocation d'investir tous les champs d'action de l'homme : les mœurs, le langage, les savoirs, les arts, les idées et les pratiques politiques, etc. ${ }^{34}$. De toutes les expressions littéraires émanant de cette culture, l'écriture épistolaire est sans doute la forme la plus flexible et la plus maniable, celle qui a permis certainement une plus large diffusion sociale. Elle représente aujourd'hui, pour les chercheurs de tous horizons, un vaste champ d'exploration encore très ouvert.

Maria Cristina Panzera

EA 4593 CLARE

Université Bordeaux Montaigne

Cristina.Panzera@u-bordeaux-montaigne.fr

\begin{abstract}
Résumé
L'essor de l'art épistolaire est l'un des aspects du renouveau culturel qui caractérise l'époque humaniste et renaissante. On connaît l'importance de la contribution apportée par Pétrarque à cette nouvelle valorisation de l'écriture épistolaire : nous étudions en particulier le thème de la gloire littéraire dans ses lettres familières par rapport au modèle de Cicéron (notamment le discours Pro Archia). L'enseignement humaniste a su traduire cet idéal intellectuel en un projet éducatif à part entière qui visait, par-delà les aspects rhétoriques de la communication épistolaire, la définition des liens interpersonnels et des codes de comportement en société.
\end{abstract}

\title{
Mots-clés
}

Épistolographie, rhétorique, Pétrarque, écoles humanistes, écriture et société.

\section{Abstract}

The development of letter writing is considered as one of the main aspects of the humanistic cultural renewal. A well-established point is Petrarch's contribution to the epistolary tradition. In this paper we study in particular in his letters the idea of the writer's fame which is based on Cicero's model (in particular the discourse Pro Archia). Humanistic schools succeeded in transforming this idealistic view into a real educational program in order to define not only rhetorical skills adapted to letters writing, but also the system of rules and conventions that regulated social behaviour.

\section{Keywords}

Epistolography, rhetoric, Petrarch, humanist schools, writing and society.

34 F. Rico, Le rêve de l'humanisme, Paris, Les Belles Lettres, 2002, p. 53-55. 